Revue des patrimoines

\title{
La petite maison dans les abattis ou l'art de rédiger aux bois par Jean Antoine de Brûletout, chevalier de Préfontaine dans son habitation de la France équinoxiale (1754-1763)
}

Emilie d'Orgeix et Céline Frémaux

\section{(2) OpenEdition}

Journals

Édition électronique

URL : http://journals.openedition.org/insitu/10338

DOI : $10.4000 /$ insitu. 10338

ISSN : 1630-7305

Éditeur

Ministère de la culture

Référence électronique

Emilie d'Orgeix et Céline Frémaux, « La petite maison dans les abattis ou l'art de rédiger aux bois par Jean Antoine de Brûletout, chevalier de Préfontaine dans son habitation de la France équinoxiale

(1754-1763) », In Situ [En ligne], 21 | 2013, mis en ligne le 12 juillet 2013, consulté le 03 mai 2019. URL : http://journals.openedition.org/insitu/10338; DOI : 10.4000/insitu.10338

Ce document a été généré automatiquement le 3 mai 2019.

\section{(†)

In Situ Revues des patrimoines est mis à disposition selon les termes de la licence Creative Commons Attribution - Pas d'Utilisation Commerciale - Pas de Modification 4.0 International. 


\title{
La petite maison dans les abattis ou l'art de rédiger aux bois par Jean Antoine de Brûletout, chevalier de Préfontaine dans son habitation de la France équinoxiale (1754-1763)
}

\author{
Emilie d'Orgeix et Céline Frémaux
}

\section{Introduction}

1 La littérature consacrée à l'architecture rurale dans les anciennes colonies françaises est rare. L'unique exemple de livre d'architecture en terrain colonial est l'ouvrage rédigé par d'Albaret intitulé « Differens projets relatifs au climat et La manière La plus convenable de bâtir dans Les pays chauds, et plus particulièrement dans les Indes occidentales » publié à Paris en $1776^{1}$. Néanmoins, à l'instar des travaux contemporains de Ledoux pour la bergerie à la Roche-Bernard, cette œuvre luxueuse, qui propose des compositions monumentales inspirées des modèles de maisons aux champs d'Androuet du Cerceau, s'avère mal adaptée au contexte et aux exigences de l'économie coloniale. C'est un ouvrage séduisant par son iconographie mais qui n’apporte pas de réelles solutions sur le terrain (fig. $\left.\mathbf{n}^{\circ} \mathbf{1}\right)\left(\right.$ fig. $\left.\mathbf{n}^{\circ} \mathbf{2}\right)$. 
Figure 1

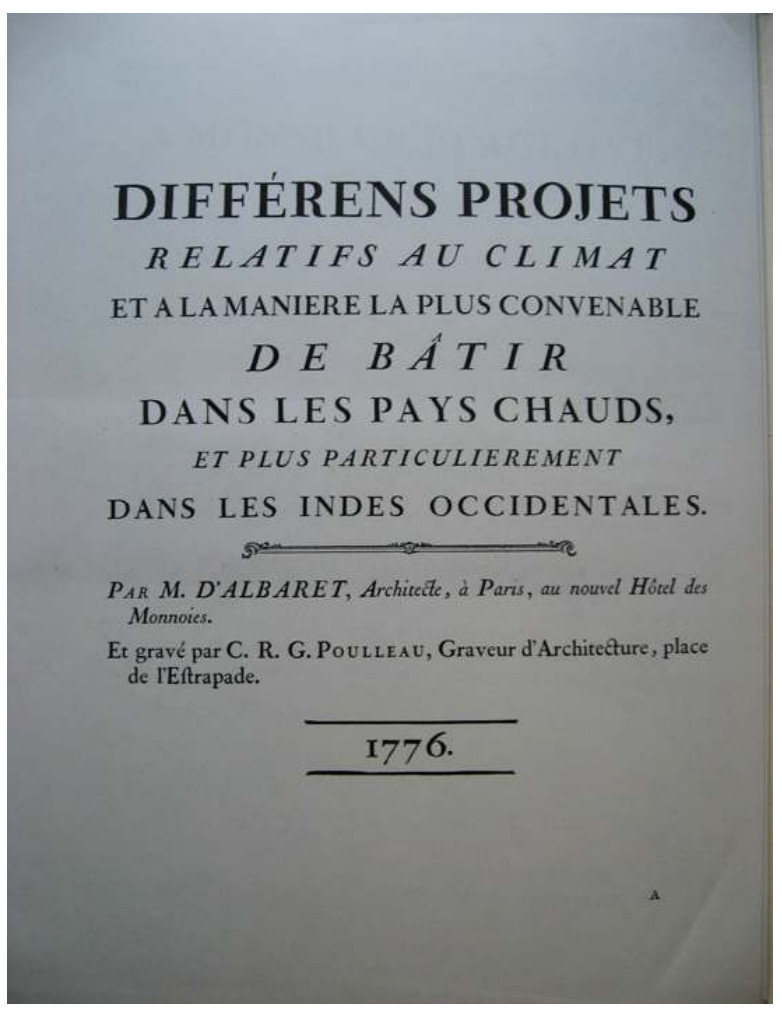

Page titre de l'ouvrage du chevalier d'Albaret. Differens projets relatifs au climat et La manière La plus convenable de bâtir dans Les pays chauds, et plus particulièrement dans les Indes occidentales. Paris, [s.e], 1776

(c) Collection particulière. 
Figure 2

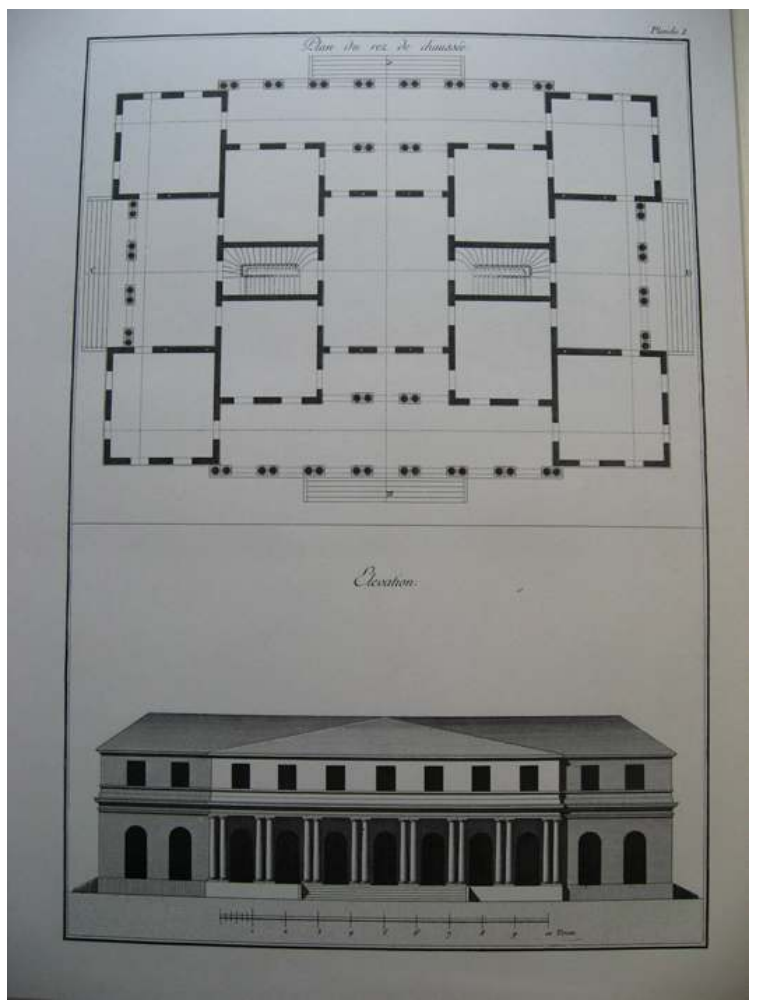

Planche 1 de l'ouvrage du chevalier d'Albaret. Differens projets relatifs au climat et La manière La plus convenable de bâtir dans Les pays chauds, et plus particulièrement dans les Indes occidentales. Paris, [s.e], 1776.

(c) Collection particulière.

2 Bien qu'il n'existe pas de traité fondateur pour la construction coloniale aux champs, de nombreuses informations peuvent être glanées dans des ouvrages en marge du livre d'architecture. Ce sont, au premier chef, des récits de voyages, tels ceux des pères JeanBaptiste du Tertre ${ }^{2}$ et Jean-Baptiste Labat ${ }^{3}$ dans les Antilles, dont les textes et les planches décrivent avec précision les constructions rurales. Ce sont ensuite les nombreux traités de botanistes suscités par les travaux de l'Académie des Sciences tel, par exemple, celui de Jean-Baptiste Thibault de Chanvallon qui sera abordé plus loin dans cet article.

3 De fait, l'architecture rurale coloniale trace plutôt son sillon dans le champ de l'économie agronome ou de l'« économie générale de la campagne » avec des titres qui reprennent souvent ceux d'auteurs français comme c'est le cas de la Maison rustique, publié à Paris par Jean Antoine de Brûletout, chevalier de Préfontaine chez Jean Sébastien Claude Bauche en $1763^{4}$ (fig. $\mathbf{n}^{\circ} \mathbf{3}$ ). 


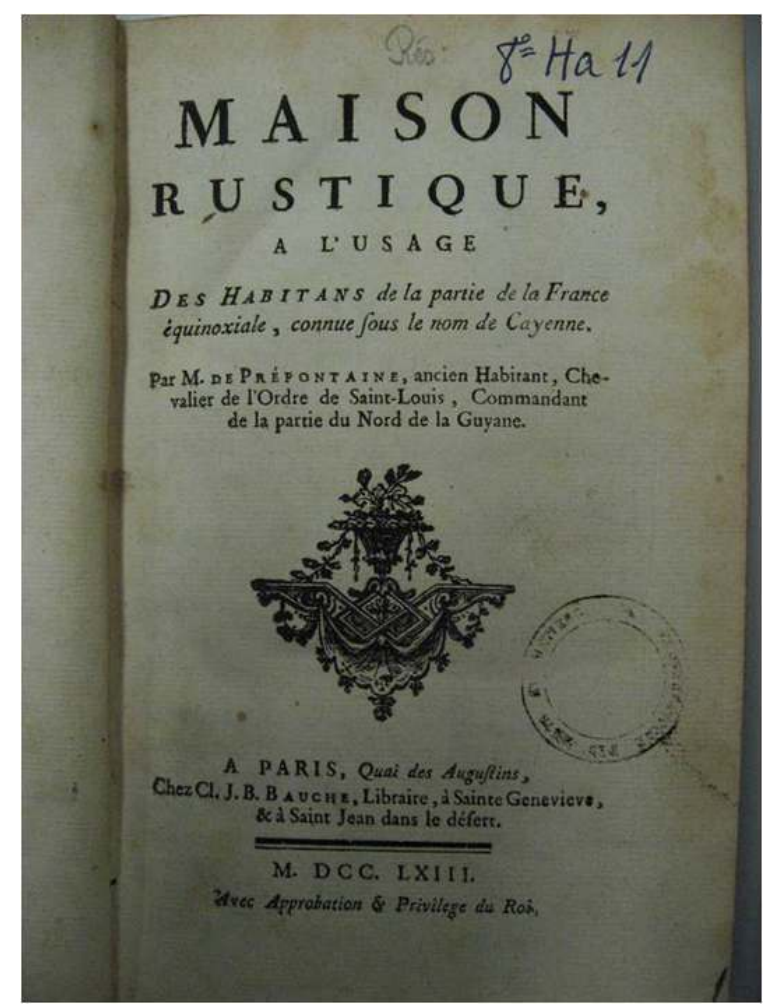

Page titre de l'ouvrage de Jean de Brûletout, chevalier de Préfontaine. Maison rustique à l'usage des habitans de la partie de la France equinoxiale, connue sous le nom de Cayenne. Paris : Bauche, 1763.

(c) Bibliothèque du CNAM, rés $8^{\circ} \mathrm{HA} 11$.

4 Faisant ouvertement référence au Praedium Rusticum ou Maison Rustique de Charles Estienne et à La Nouvelle maison rustique de Louis Liger respectivement publiés en 1554 (en latin), 1564 (en français), et 1700, la nouvelle Maison rustique s'inscrit dans cette lignée d'ouvrages d'agronomie, à une période où les livres traitant spécifiquement d'architecture "champêtre" suivant l'expression de Jacques François Blondel ${ }^{5}$, sont encore rares. À première vue, il paraît s'agir de l'œuvre désintéressée d'un propriétaire local, « épris d'un zèle tout patriotique » pour inciter de nouveaux aventureux à s'établir en Guyane et faciliter leur adaptation au terrain. Néanmoins, la relative transparence de son ambition prend un jour nouveau une fois transposée dans la société interlope et mercantiliste de la France équinoxiale du milieu du XVIII ${ }^{\mathrm{e}}$ siècle.

5 L'ouvrage de Préfontaine se prête à deux lectures successives. La première analyse sa contribution du point de vue des pratiques agricoles et architecturales de la colonie. C'est-à-dire qu'elle s'arrête sur la vision riante et champêtre que Préfontaine voulait transmettre à son public d'élection. La seconde aborde, à travers l'étude du projet éditorial, du choix de l'éditeur et du contexte politique et scientifique très particulier de l'époque et de la colonie, les ambitions sous-jacentes de la Maison rustique, qui loin d'être uniquement un ouvrage d'agronomie coloniale se révèle être l'instrument majeur d'un vaste projet d'ascension politique, sociale et économique. 


\section{Préfontaine et son « habitation ${ }^{6}$}

6 Le personnage de Jean Antoine de Brûletout, sieur de Préfontaine, illustre parfaitement le parcours d'un bourgeois aventurier du XVII ${ }^{e}$ siècle qui a su tirer opportunité du système mis en place par l'administration coloniale. Né en 1717 à Paris, il est le fils d'Antoine Brûletout, bourgeois de Paris et de Marie de Préfontaine. Envoyé dans la colonie à la fin des années 1730 comme lieutenant de la Marine, il est ensuite lieutenant de la garnison de Cayenne en 1746, date de son premier mariage. Préfontaine est déjà dans les années 1750 un personnage influent de la colonie, comme le démontre la grande carte de l'île de Cayenne datée de cette période qui détaille sa poursuite et sa « découverte » des nègres marrons, esclaves en fuite réfugiés dans la forêt ${ }^{7}\left(\right.$ fig. $\left.^{\circ}{ }^{\circ}\right)$ ). Le détail de cette expédition, mise au propre par Louis Charles Buache, est publié dans une carte «dressée sur les observations estimées et les remarques de Mr. De Préfontaine, capitaine d'infanterie » datée de $1762^{8}$.

Figure 4

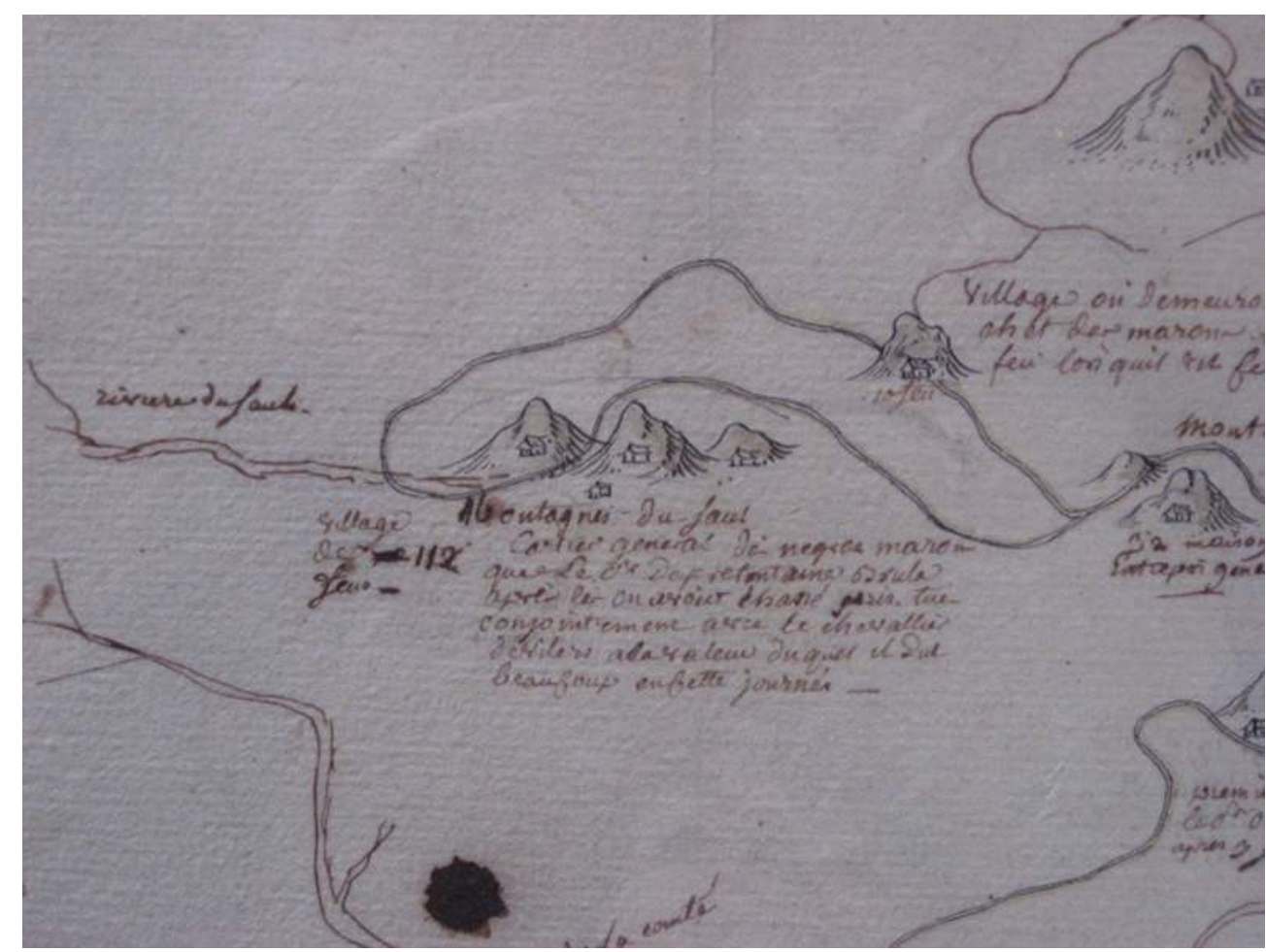

Détail de la carte manuscrite de Guyane mentionnant les opérations menées par le Sieur de Préfontaine en 1750 pour « rattraper les nègres marrons », vers 1750-60.

(c) BnF, département des Cartes et Plans, Rés Ge B 1172.

7 La seconde épouse de Préfontaine, avec laquelle il s'unit en 1752, héritière de nombreux terrains à Macouria, élève son statut de soldat milicien à celui d'« habitant propriétaire ». Ce mariage lui apporte notamment une sucrerie de 62 esclaves et une «ménagerie » de 120 têtes de bétail (fig. $\mathbf{n}^{\circ}$ 5). C'est à cette époque (1754) que, mis aux arrêts pour insubordination pour des motifs restés obscurs, il se consacre pleinement à l'aménagement de sa nouvelle propriété au lieu-dit «La Félicité » dans le quartier de Macouria. C'est durant la décennie 1750-1760 qu'il rédige son imposant ouvrage dédié à 
l'attention des futurs propriétaires terriens de Guyane et plus largement «dans les colonies en général ».

Figure 5

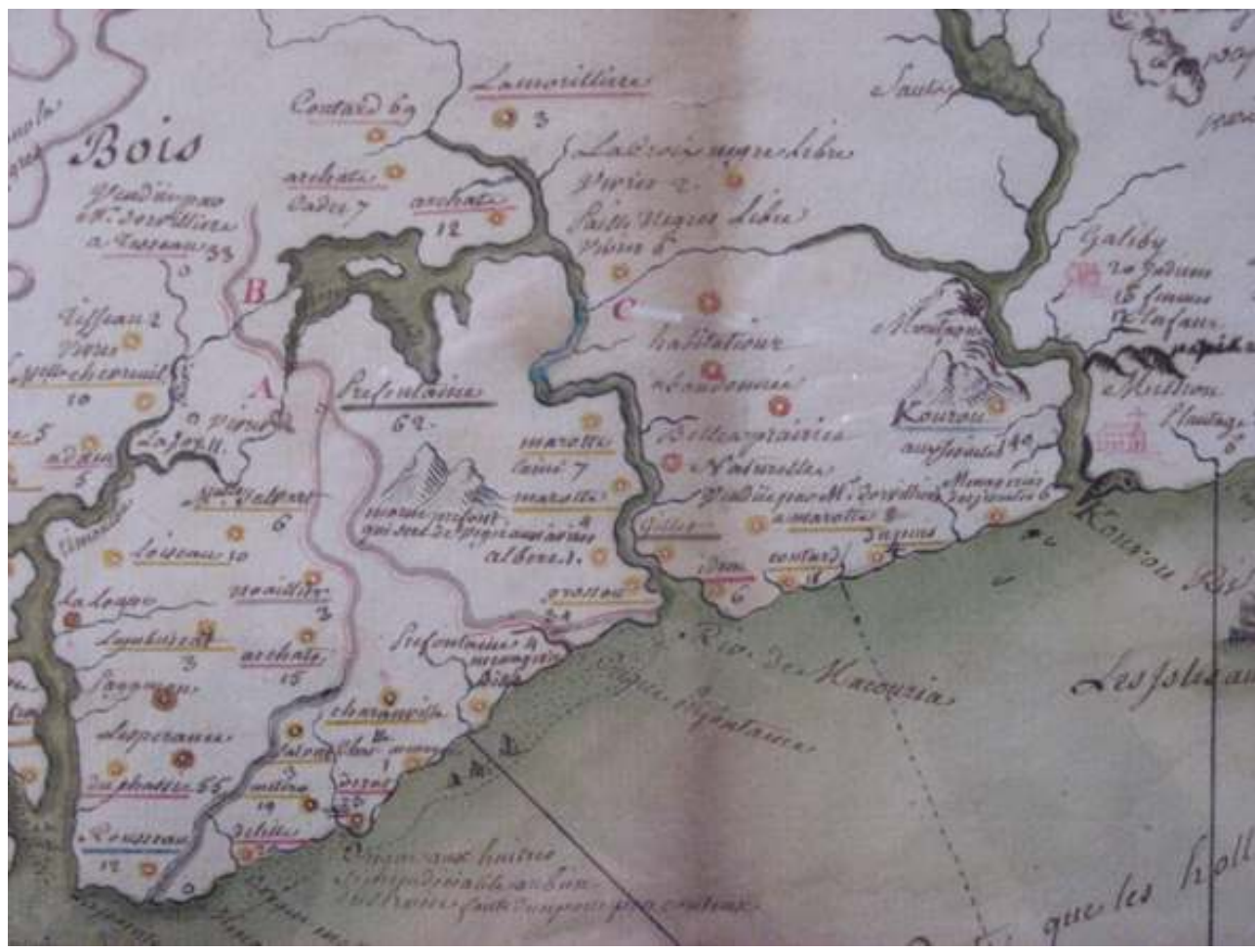

Détail de la carte manuscrite de Guyane mentionnant l'emplacement de l'habitation du Sieur de Préfontaine (coté 62) vers 1750-60

(c) BnF, département des Cartes et Plans, Rés Ge B 1172.

\section{La vie rustique dans une " habitation » selon Préfontaine}

$8 \quad$ La Maison rustique de Préfontaine traite de tous les aspects de la vie quotidienne dans une exploitation coloniale rurale. Les trois premiers chapitres de l'ouvrage, en particulier, concernent le défrichement des lieux par abattis (coupe du bois puis brûlis de la parcelle) et la construction de la maison de maître avec ses différents bâtiments d'exploitation. L'iconographie, composée de sept planches en taille-douce gravées par Nicolas Chalmandrier, graveur de géographie, illustre principalement ces premiers chapitres. Quatre des sept planches détaillent l'habitation incluant le logement et les dépendances de la maison coloniale aux champs.

9 Les chapitres restants sont consacrés aux différentes plantes utilisées dans les manufactures (tabac, riz, café, coton, rocou), à l'organisation du travail des esclaves et aux travaux de défrichage. Les chapitres concernant l'histoire de la flore sont largement inspirés de travaux contemporains dont celui de Charles Plumier "Description des plantes de l'Amérique » (1693), de Georg Marcgrave « Histoire naturelle du Brésil » (1648) et de Jacques François Arthur, "Histoire des colonies françoises de la Guyane » (1753-1768). Néanmoins, l'œuvre de Préfontaine n'est pas uniquement le fruit de 
compilations. Son chapitre sur le rocou est ainsi inédit et découle directement de son expérience du terrain guyanais.

Selon Préfontaine, la première action que doit mener tout habitant-concessionnaire est de choisir judicieusement l'emplacement de sa maison sur son terrain. Il rappelle les principes énoncés par Vitruve, suggérant que le terrain où elle sera construite doit être consciencieusement évalué en fonction des vents et d'une situation surélevée. «Un petit plateau ou l'on monterait par une pente douce et qui dominerait sur toutes les dépendances de la maison doit être préféré » (p. 7.). Dans le cas des colonies, la situation de commande du terrain n'a pas uniquement pour vocation la salubrité mais aussi la surveillance des esclaves par leurs propriétaires. "Tous les autres bâtiments qui regardent l'économie intérieure, ainsi que les cases des nègres et ceux qui concernent la manufacture à laquelle on s'adonne doivent être sous les yeux du maitre. Ceux qui tient au ménage comme magasin à vivre et cuisines doivent être vue de la maitresse de l'endroit où elle travaille». Le détail de l'organisation spatiale de l'habitation est accompagné d'une planche décrivant prétendument la luxueuse maison de Préfontaine (fig. $\left.\mathbf{n}^{\circ} 6\right)\left(\right.$ fig. $\left.n^{\circ} 7\right)$.

Figure 6

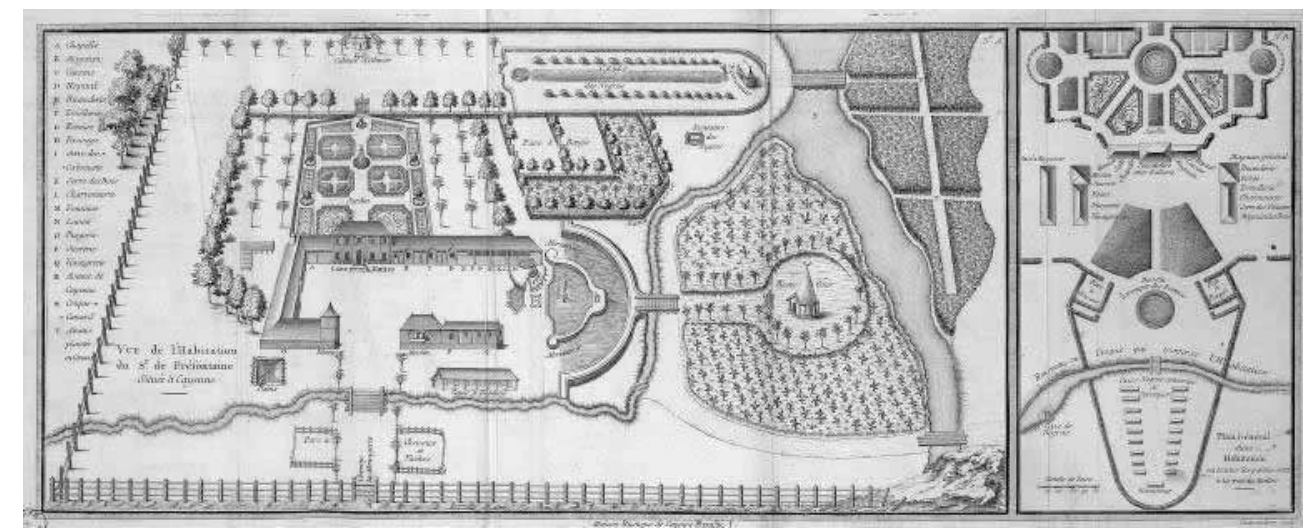

Détail de la planche 1 illustrant l'habitation modèle proposée par Jean de Brûletout, chevalier de Préfontaine. Maison rustique à l'usage des habitans de la partie de la France equinoxiale, connue sous le nom de Cayenne. Paris : Bauche, 1763.

(c) Bibliothèque du CNAM, rés $8^{\circ} \mathrm{HA} 11$. 
Figure 7

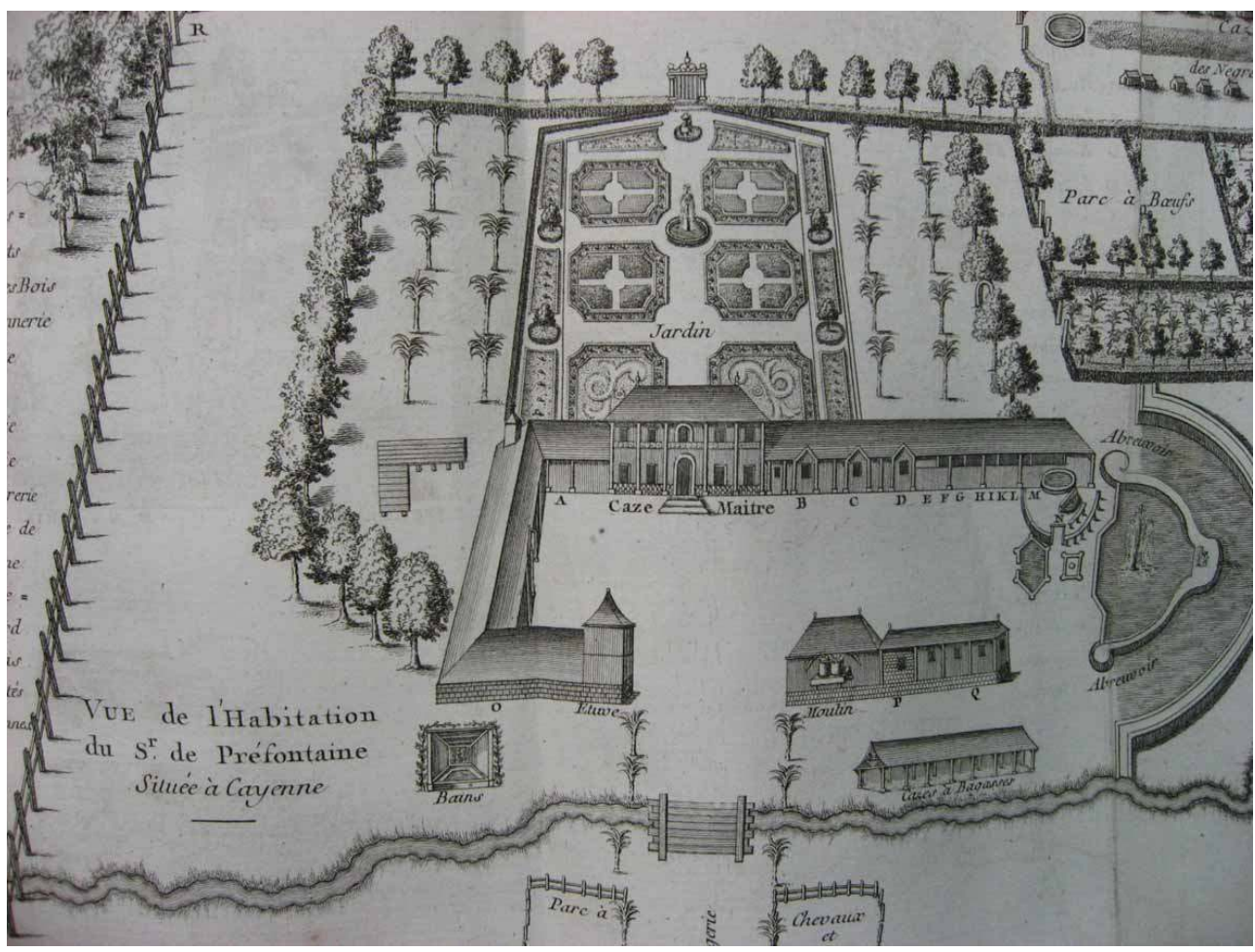

Détail de la planche 1 illustrant l'habitation modèle proposée par Jean de Brûletout, chevalier de Préfontaine. Maison rustique à l'usage des habitans de la partie de la France equinoxiale, connue sous le nom de Cayenne. Paris : Bauche, 1763.

(c) Bibliothèque du CNAM, rés $8^{\circ} \mathrm{HA} 11$.

11 Le mode de construction des bâtiments est ensuite précisément détaillé dans un troisième chapitre. Il révèle ici l'influence de l'architecture amérindienne, notamment la case des indiens Galibis appelée carbet. Selon Préfontaine, les habitations rurales sont rarement élevées sur plusieurs niveaux. Pitou dans son voyage en Guyane de 1777 commente d'ailleurs ce fait en notant que même à Cayenne, les maisons à deux étages «sont des palais ${ }^{9}$ (fig. $\left.\mathbf{n}^{\circ} \mathbf{8}\right)$. 


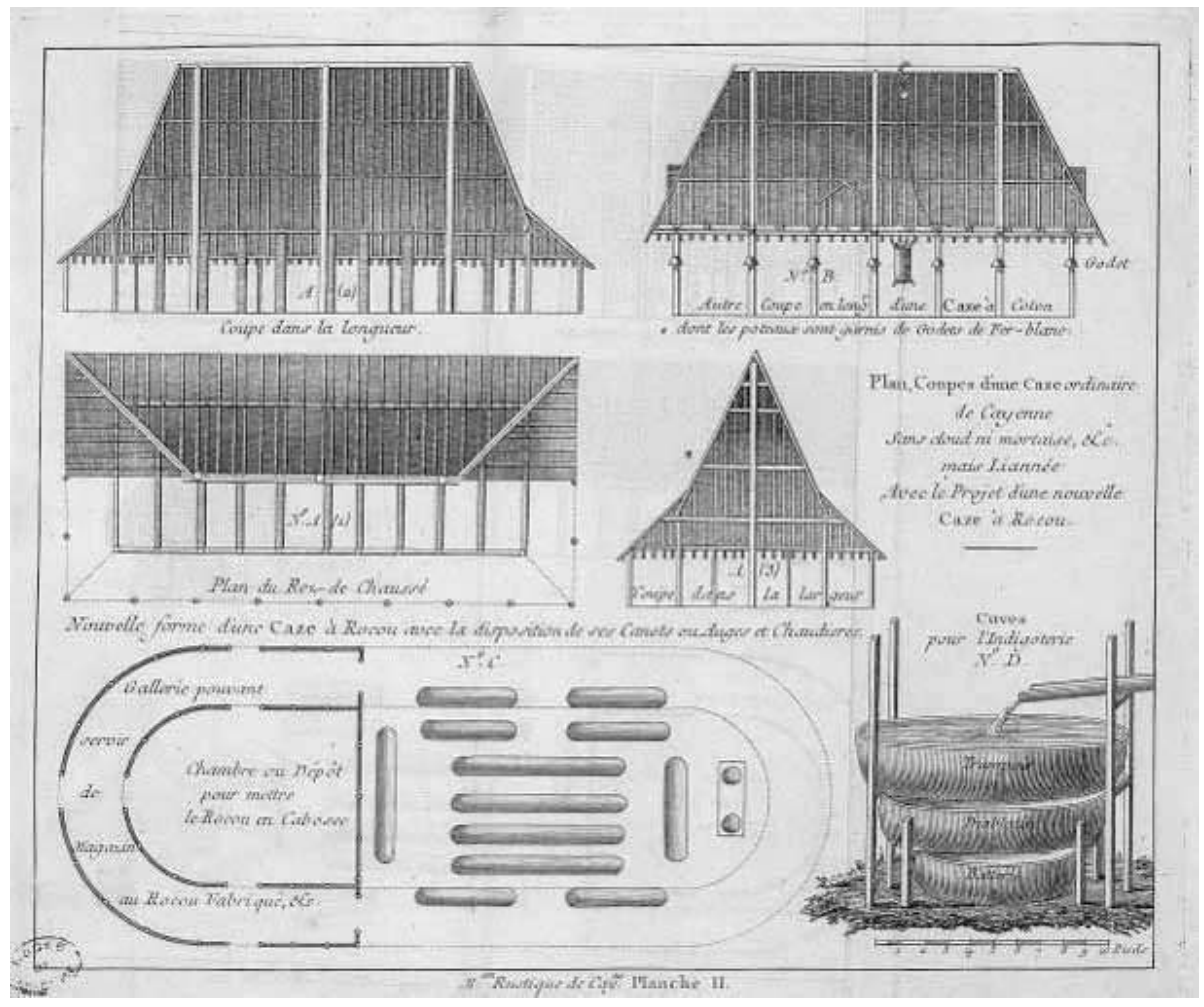

Détail de la planche 6 illustrant un plan et profil d'une case indigène proposée par Jean de Brûletout, chevalier de Préfontaine. Maison rustique à l'usage des habitans de la partie de la France equinoxiale, connue sous le nom de Cayenne. Paris : Bauche, 1763.

(c) Bibliothèque du CNAM, rés $8^{\circ} \mathrm{HA} 11$.

Le logement des maîtres est rectangulaire. Les bâtiments comprennent une pièce centrale et deux chambres aux extrémités. Ils sont entourés et protégés par une profonde galerie ouverte et constitués d'une simple structure de poteaux de bois plantés dans le sol «en fourche » sans tenons, mortaises ni chevillages, mais «liannés » entre eux. Les espaces sont ensuite remplis de tançons de bois sec avant d'être "bousillés » avec de l'argile parfois mêlée de paille. Les bâtiments sont ensuite couverts de simples toitures de feuilles de cannes ou de roseaux. Les mêmes modes de construction prévalent pour les autres dépendances de l'habitation hormis la cuisine, toujours séparée de la maison principale, et qui est la seule dépendance partiellement construite de pierre en utilisant généralement la pierre à ravets : " une roche trouée, rougeâtre, incapable de soutenir aucun ornements d'architecture mais qui sert aux ouvrages solides ». La construction des dépendances est également détaillée dans l'ouvrage. Préfontaine souligne qu'il est avantageux pour un habitant de construire une basse-cour sur son habitation. « La forme ronde en pain de sucre est plus avantageuse en ce qu'elle donne moins prise au vent. Dedans, il doit y avoir un petit poulailler séparé où on traite la volaille qu'on donne aux nègres ou aux indiens. Au devant, il doit $\mathrm{y}$ avoir une galerie large et basse pour que la petite volaille puisse s'y promener à l'abri du vent » (p. 22).

Les fouilles archéologiques et les études menées sur les habitations de la période coloniale en Guyane ${ }^{10}$ corroborent l'usage de ces modes de construction. Les liens avec l'habitation rurale en France, notamment par la pérennité des modèles, l'utilisation des matériaux locaux, l'adaptation aux techniques locales - structures liannées, supports en fourches en 
terre, couvertures de feuilles, bousillage, cheminées dans souches - sont d'ailleurs assez marquants.

On est loin ici du contexte florissant de la colonie voisine de Saint-Domingue qui faisait écrire à Moreau de Saint Méry en 1789 que «le luxe y avait un culte très suivi ${ }^{11}$ ». En Guyane, il faut attendre la fin du XVIII ${ }^{e}$ siècle pour que commencent à apparaître quelques matériaux et ornements importés par bateau, notamment des éléments de ferronnerie, de la chaux, des dalles de schiste et de marbre. L'unique exemple d'importation massive est la tentative d'importation en 1766 par l'entreprise Fiedmond \& Maillard Dumesle de quelques maisons préfabriquées en bardeau de sapin depuis la nouvelle Angleterre ${ }^{12}$. Mais pour l'ingénieur suisse Guisan, chargé de la mise en valeur des terres basses de Guyane, auteur du Traité sur les terres noyées de Guyane en 1788, les meilleures constructions sont celles visant à ménager un espace de vie commode et sain, et bannissant tout luxe « ridicule et inutile dans un établissement de culture ${ }^{13}$ ».

Malgré le décalage évident entre texte et iconographie, cette première lecture de l'œuvre de Préfontaine livre donc une vision sereine de la vie des habitants de la colonie centrée autour de leur habitation riche en possibilités d'exploitation, fertile, et à la faune et la flore abondantes. Elle semble préfigurer, du point de vue éditorial, une sorte de " protomanuel » du propriétaire terrien, genre qui deviendra florissant en France au siècle suivant.

Ce n'est qu'à la lumière des sources contemporaines que la réalité du terrain apparaît sous un jour nouveau et que la construction pérenne de «la maison aux champs » telle que Préfontaine la décrit dans ses planches s'avère bien plus complexe qu'il ne la dépeint.

17 En effet, Préfontaine, pourtant aguerri aux vicissitudes de la colonie, ne fait qu'effleurer la complexité du terrain guyanais. Colonie très différente des Antilles qui disposaient de riches sols volcaniques, la Guyane possède dans ses terres hautes (c'est-à-dire non inondées contrairement aux terres basses) des sols d'origine cristalline qui s'épuisaient très rapidement. La technique de l'abattis ou de l'agriculture itinérante sur brûlis, auquel Préfontaine dédie son premier chapitre était le moyen quasi exclusif d'exploitation des terres. Comme l'avait déjà souligné quelques décennies plus tôt Goupy des Marets, observateur avisé de l'agriculture en Guyane: «Lorsque l'on s'est établi sur une terre femelle, où l'on est assuré qu'au bout de 3 ans il faudra la quitter pour s'établir dans un autre endroit afin de s'approcher des cannes que vous avez posées et plantées dans de nouveaux abattis, il faut se résoudre à ne faire que des bâtiments de bousillage et à peu de frais afin de les pouvoir laisser lors qu'on abandonne un vieux terrain ${ }^{14} »$. Les gouverneurs de la colonie, dont d'Orvilliers et Lemoine, se plaignent d'ailleurs sans relâche du nomadisme et du laisser aller des habitants de la colonie qui sont devenus, à l'exemple des indiens, eux-mêmes ambulants ${ }^{15}$ : «Un abattis de rocou rapporte 4 à 5 ans, les bâtiments ne sont construits que pour ce temps. L'habitant suit son abattis et promène ses bâtiments dans toutes les parties de son habitation. Des pieux plantés et clissés forment le corps du bâtiment. La première feuille indifféremment tombée forme la couverture et cela lui suffit. Le colon prétend même qu'il n'existe aucune autre solution et que c'est l'expérience qui l'a instruit ${ }^{16} »$.

De fait, la riante habitation dépeinte dans l'ouvrage de Préfontaine semble tout à fait fictive si l'on considère les conditions d'établissement dans la colonie au milieu du XVIII siècle. Il faut noter ici que la Guyane ne comprend alors que trente-quatre habitations sur l'ensemble de son vaste territoire. Le décalage avec la colonie voisine hollandaise du Surinam fait d'ailleurs rêver l'intendant Pierre Victor Malouet de changer de nationalité. 
«J'aimais fort » dit-il «trouver en débarquant sur une habitation [du Surinam], un quai propre et commode et un chemin carrelé au moyen duquel j'arrivais sans m'embourber à la maison du maitre. Je louais aussi mon hôte d'avoir un joli jardin bien garni d'arbres fruitiers, de légumes et une basse-cour abondamment fournie. En comparant tout cela à la mesquinerie, à la malpropreté et à la misère des habitations de Cayenne, j'étais bien tenté de me faire adopter par les Hollandais et de renoncer tout bon à la France équinoxiale ${ }^{17}$ ".

L'une des tentatives qui fut employée pour améliorer l'état de la colonie - mais ce n'est pas ici l'objet de cette étude - fut la création d'« habitations royales ", qui, à l'instar des fermes modèles, avaient pour vocation d'être des " habitations écoles ", dont "L'épicerie de La Gabrielle », la « ménagerie du Roy à Sinnamary », «l'habitation du Roy à Cayenne ». Elles fonctionneront un peu plus tard, après 1775 . Mais, tandis qu'elles devaient servir de modèles par l'introduction de nouvelles épices (tels les girofliers à La Gabrielle), la mise au point de techniques agraires dans les terres basses et la sélection du bétail, elles n'avaient qu'un rendement médiocre et coûtaient fort cher à la colonie. En outre, les maisons de maître de ces habitations royales n'étaient pas plus fastueuses que les habitations privées. Celle de La Gabrielle, habitation régie par le sobre Guisan, n'était, d'après l'inventaire de 1790, qu' « une mauvaise case fourches en terre, d'environ cinquante pieds de long sur vingt pieds de large couverte de mauvais bardeau de carapa, servant d'économat revêtu des quatre côtés en planches d'angélique. Comportant quatre chambres de plein pied $^{18}$.»

\section{Les ambitions cachées : le voyage en France et l'édition du traité}

Le décalage entre le discours de La Maison rustique et la réalité coloniale ouvre la voie à une seconde lecture critique de l'ouvrage de Préfontaine. Si son lyrisme agricole n'était pas dû aux joies de sa récente union avec une riche héritière, son œuvre devait certainement suivre un fil directeur différent, fil que nous avons tenté de démêler sur d'autres rivages.

Pourquoi choisir d'imprimer à Paris chez Jean Baptiste Claude Bauche second ? L'éditeur imprimeur parisien connu pour tenir boutique au quai des Augustins, à l'image SainteGeneviève, est alors en bien mauvaise posture. Il connaît de nombreuses difficultés financières et est sur le point de vendre son fonds de librairie, ce qu'il fera dans la décennie 1760, avant de déclarer faillite en $1772^{19}$. Le choix de Préfontaine ne semblerait donc pas judicieux si l'on ne notait dans le catalogue de Bauche plusieurs autres ouvrages, datés de la même décennie, consacrés aux colonies et tous dédiés, comme celui de Préfontaine, au marquis de Choiseul, ministre de la Marine et ancien ministre des Affaires étrangères. Un de ces ouvrages est notamment le Voyage à la Martinique de Jean Baptiste Thibaut de Chanvallon (Paris, 1763) qui avait été lu en 1761 à l'Académie des sciences (fig. $\mathbf{n}^{\circ}$ 9). Or, Thibaut de Chanvallon, botaniste colonial, est également un fin politicien, proche condisciple de Préfontaine et actif en Guyane où il est nommé intendant de Cayenne en 1763. C'est également un proche de Choiseul, qui prépare un vaste projet de colonisation en Guyane après la perte de la Nouvelle-France en février 1763 lors du traité de Paris. 
Figure 9

\author{
V O Y A G E \\ A LA MARTINIQUE, \\ C O N T N A N T \\ DIVERSES Obfervations fur la Phyfique, \\ l'Hiftoire Naturelle, l'Agriculture, les Maurs, \\ o les Ujages de cette Ifle, faites on 1751 or \\ dans les années fuivantes. \\ Lu a l'Académie Royale des Sciences de Paris en $176 x$.

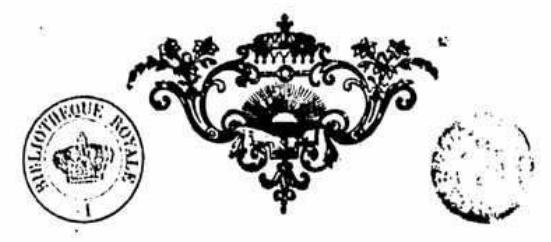 \\ A PARIS, Quay des Auguftins,
Chez Cl. J. B. B $A$ v crz, Libraire, a Sainte Generieve,
\& a Saint Jean dans le deffert.

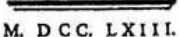 \\ AVEC APPROBATION ET PRIVILEGE DU ROI.
}

Page titre de l'ouvrage de Jean Baptiste Thibaut de Chanvallon. Voyage à la Martinique : contenant diverses observations sur la physique, l'histoire naturelle, l'agriculture, les mœurs, \& les usages de cette isle, faites en 1751 \& dans les années suivantes. Paris : Jean-Baptiste Bauche, 1763.

C'est à la lumière de ces considérations que le manuscrit de Préfontaine prend enfin tout son sens politique. Présentée à Choiseul en 1762 et approuvée pour publication, La maison rustique devait servir de vitrine officielle pour favoriser l'émigration en Guyane dans le cadre des nouveaux efforts de colonisation.

Préfontaine évolue donc à Paris dans le cercle des physiocrates proches de Choiseul, notamment le marquis de Bombarde, un parent de Choiseul, dont l'ambition était de créer une colonie blanche. C'est lui qui initia la désastreuse fondation de Bombardopolis dans le centre de Saint-Domingue entre 1764 et 1768 (fig. nº $^{\circ}$ ). 


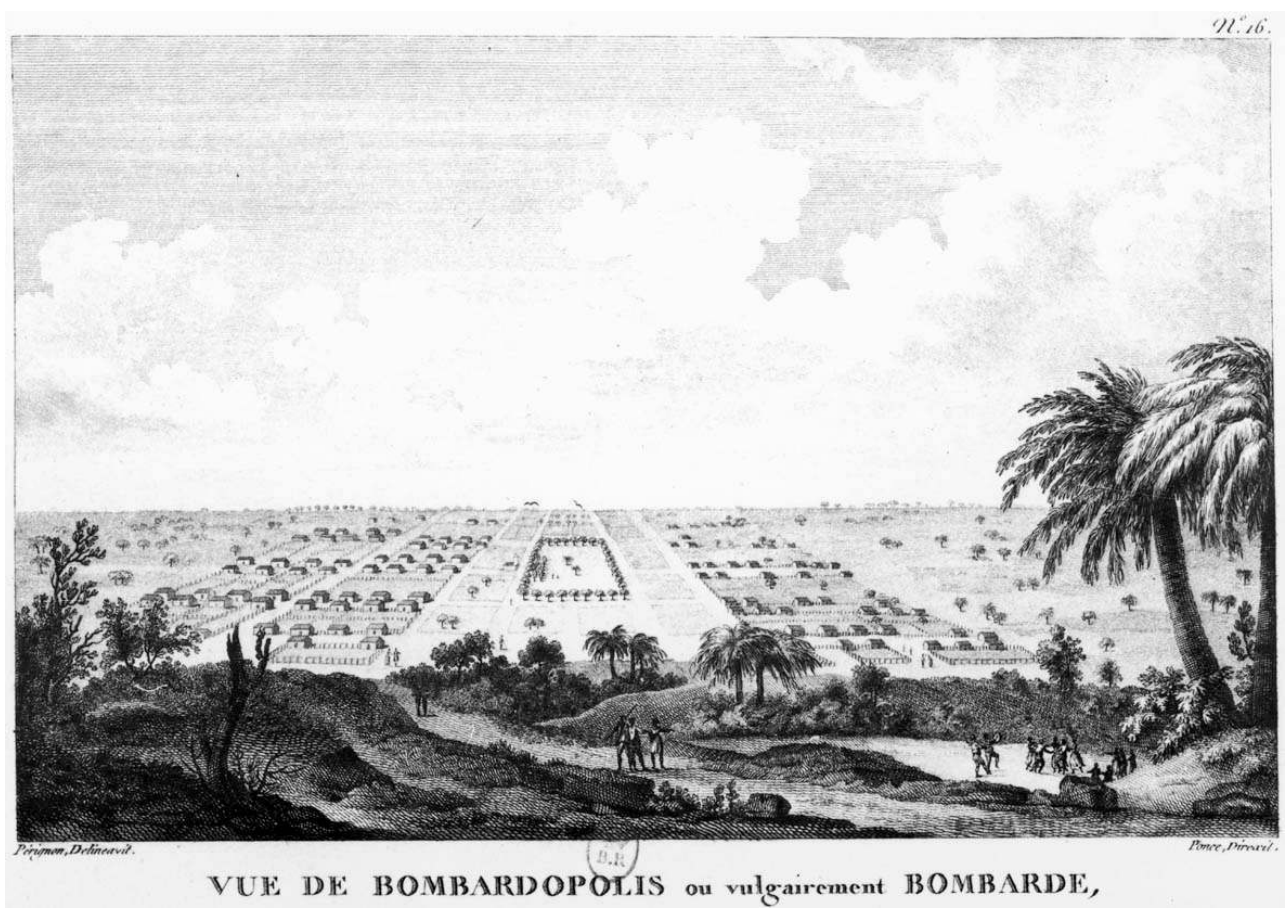

Nicolas Ponce. Vue de Bombardopolis ou vulgairement Bombarde. Isle St. Domingue. Paris, 1791.

(c) BnF, Réserve F 1901 R 311795 Folio.

Ainsi, contrairement aux allégations de Préfontaine qui s'avouait dans sa préface «tout étonné d'avoir fait un livre ", son manuel pratique du propriétaire-concessionnaire était une œuvre de stratégie sociale mûrement réfléchie. En 1763, Préfontaine succède au gouverneur d'Orvilliers. Il est nommé commandant de la partie nord de la Guyane, poste qu'il occupe de mai 1763 au 22 décembre 1764. Sa nomination s'accompagne d'un projet d'établissement de nègres marrons le long du Maroni et d'un projet de commerce à Cayenne. C'est à ce moment-là que le programme de la nouvelle colonie de Sinnamary et de Kourou est annoncé. Préfontaine est chargé d'organiser l'arrivée des premiers colons et de choisir le site. Mais alors que son projet originel était de fonder une petite colonie de 300 hommes, Choiseul décide un plan de colonisation massive. Préfontaine réalise donc des plans des sites à coloniser (fig. $\left.\mathbf{n}^{\circ} \mathbf{1 1}\right)$ (fig. $\mathbf{n}^{\circ}{ }^{\mathbf{1 2}}$ ). La suite, connue sous le nom de l'expédition de Kourou, aboutit à un désastre. Sur l'ensemble des colons qui y participèrent (12 à 15000 selon les sources), 9000 décédèrent ${ }^{20}$. Il s'ensuivit un long procès, qui aboutit dans un premier temps à de lourdes condamnations, notamment celle de Thibault de Chanvalon, chef de la première expédition, avant que les condamnés ne soient réhabilités en 1781 . 
Figure 11

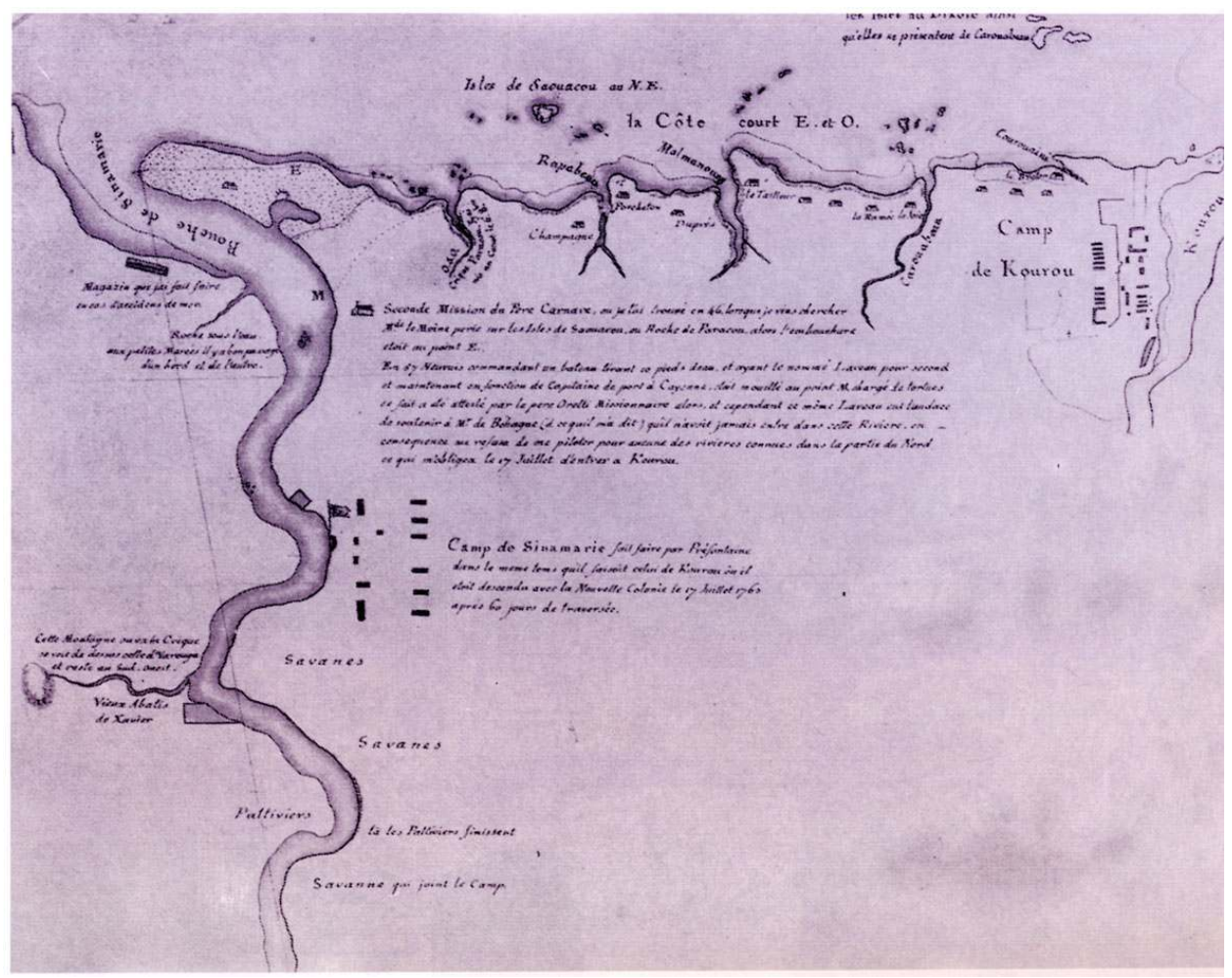

Plan de Sinamary fait faire par Préfontaine dans le même temps qu'il faisait faire celui de Kourou où il était descendu avec la nouvelle colonie le 17 juillet 1762 après 60 jours de traversée, 1763.

(c) BnF, Ce C 5003

Figure 12

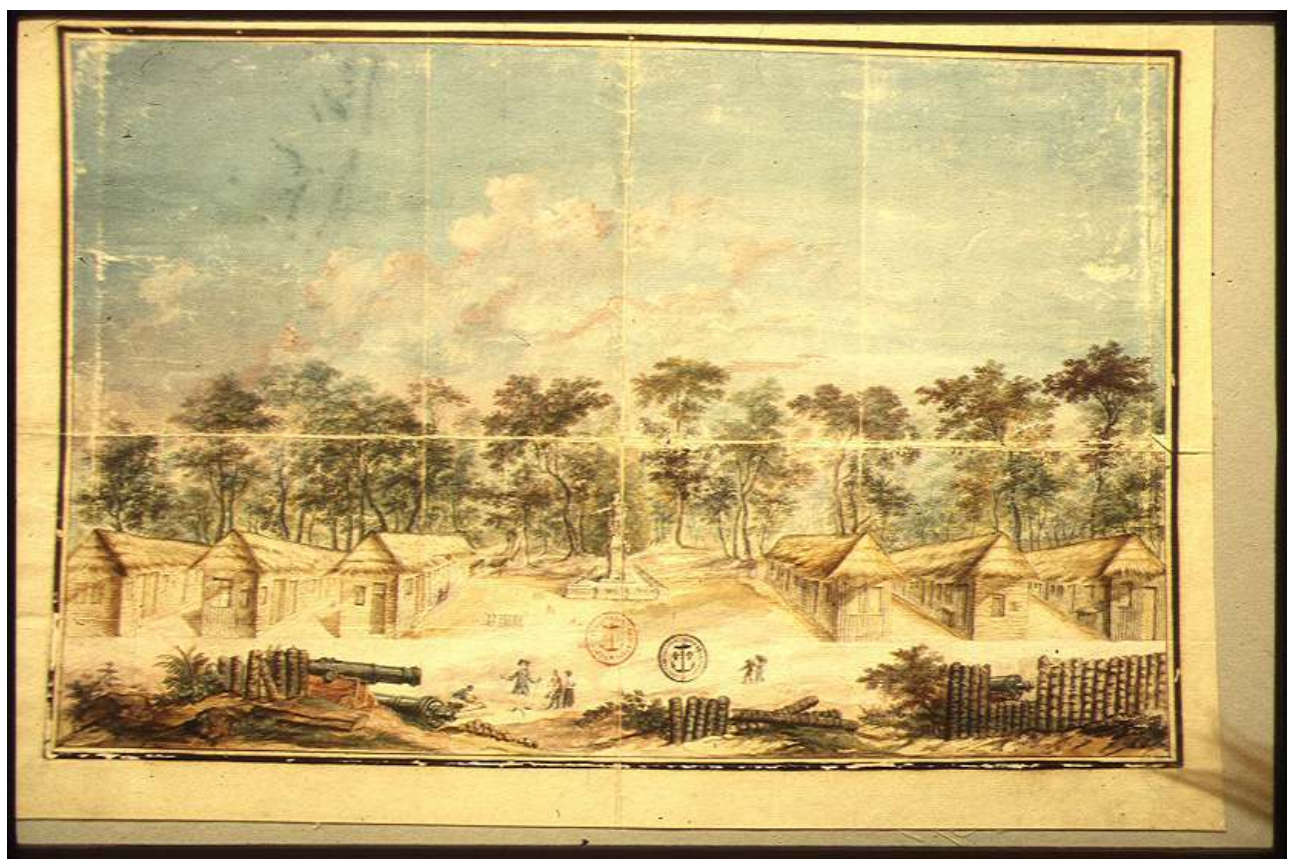

Vue de Kourou en Guyanne, 1762

(c) AN, CAOM, DFC, 21 pfb 135 
Préfontaine, blâmé et appauvri revint alors vivre dans son habitation de la Félicité à Macouria où il mourut vingt ans plus tard, en 1787.

\section{Conclusion}

Émilie d'Orgeix est maître de conférences en histoire de l'art moderne à l'université de Bordeaux 3. Ses recherches portent sur la culture architecturale civile et militaire en Europe et dans les colonies d'Amérique à l'époque moderne. emilie.dorgeix@ubordeaux3.fr

Céline Frémaux est conservateur de l'inventaire général du patrimoine culturel de la Région Guyane. Elle s'intéresse au patrimoine architectural colonial et contemporain. celine.fremaux@cr-guyane.fr

\section{NOTES}

1. - Chevalier D'ALBARET. Differens projets relatifs au climat et La manière La plus convenable de bâtir dans Les pays chauds, et plus particulièrement dans les Indes occidentales. Paris, [s.e.], 1776. 
2. - Du TERTRE, Jean- Baptiste. Histoire générale des Antilles habitées par les Français.Paris : Thomas Jolly, 4 vol., 1667-1671.

3. - LABAT, Jean-Baptiste. Nouveau voyage aux isles de l'Amérique, contenant l'histoire naturelle de ces pays, l'origine, les mœurs, la religion et le gouvernement des habitans anciens et modernes, les guerres et les événemens singuliers qui y sont arrivez... le commerce et les manufactures qui y sont établies... ouvrage enrichi de plus de cent cartes, plans et figures en taille-douce. La Haye : P. Husson, 1724, 4 vols.

4. - De BRULETOUT, Jean Antoine, chevalier de Préfontaine. Maison rustique à l'usage des habitans de la partie de la France equinoxiale, connue sous le nom de Cayenne [...]. Paris : Bauche, 1763.

5. - GARRIC, Jean-Philippe. Fermes idéales en Limousin: Architecture et agriculture au XIXe siècle. GRANDCOING, Philippe (dir) avec la collaboration d'Alain Blanchard, Nicole de Blomac, Dominique Danthieux et Jean-Philippe Garric, préface de Marion Segaud). Éditions Culture \& Patrimoine en Limousin, 2010, p. 16.

6. - Le terme habitation désigne un établissement agricole voué à la production de denrées coloniales comme le sucre, le café, le cacao, etc. Il englobe l'ensemble de l'exploitation coloniale, bâtiments et terrains.

7. - Carte de lisle de Cayenne ou l'on voit les positions de chaque habitants, la nature de sa fabrique, le nombre de ses esclaves avec la marche du Sieur de Préfontaine dans sa découverte des nègres marrons en 1747... BnF, Cartes des Plans, Rés Ge B 1172, v. 1750-1760.

8. - Carte géographique de lisle de Cayenne et de ses environs dressée sur les observations estimées et les remarques de Mr de Préfontaine, capitaine d'infanterie par Louis Charles Buache, sous les yeux de Phil. B. Per géographe de sa majesté présenté à Monseigneur Le Duc de Choiseul par l'auteur des mémoires en septembre 1762. BnF, Cartes des Plans, Ge C 5003.

9. - PITOU, Louis Ange. Voyage à Cayenne, dans les deux Amériques et chez les anthropophages. Ouvrage orné de gravures, contenant le tableau général des déportés, la vie et les causes de l'exil de l'auteur, des notions particulières sur Collot et Billaud... Par Louis-Ange Pitou. Paris : chez l'auteur, An VIII (1805), p. 59.

10. - Voir à ce sujet la thèse de Yannick LE ROUX. L'habitation guyanaise sous l'Ancien Régime. Paris : EHESS, 1994, 2 volumes.

11. - Voir l'article de Christophe Charlery. " Maisons de maître et habitations coloniales dans les anciens territoires français de l'Amérique tropicale ». In Situ, Revue des Patrimoines, ${ }^{\circ} 5$, décembre 2004, note 9. http://www.insitu.culture.fr/article.xsp? numero=5\&id_article=d4-1023\&qid=sdx_q0\#n9

12. - Archives Nationales, C 14, R 29 F 114, 1766, Maisons en bois de sap. Couverte en bardeaux de même bois.

13. - GUISAN, Samuel. Traité sur les terres noyées de la Guyane. Cayenne : imprimerie royale, 1788, p. 255.

14. - GOUPY DES MARETS. Voyage de Goupy aux Isles d'Amérique et aux côtes de l'Afrique, 1675-76 et 1687-1690. Ms. Montbret, $\mathrm{n}^{\circ} 125$, bib. de Rouen, 400, p. 178.

15. - AN, CAOM, DFC, Guyane, C6. F 339. Mémoire, 1780.

16. - D'Orvillliers et Lemoine, Mémoire sur la Guyane, AN, CAOM, DFC, Guyane, Carton $60, n^{\circ} 71$, 1761 (cité par Yannick Leroux, op. cit., p. 506).

17. - Malouet, DFC, Guyane, voyage au Surinam, C 63 F³05, 1777 (cité par Yannick Leroux, op. cit., p. 557).

18. - Cité par Yannick Le Roux, op.cit., p. 539.

19. - BARBIER, Frédéric, JURATIC, Sabine, MELLERIO, Annick. Dictionnaire des imprimeurs, libraires et gens du livre à Paris (1701-1789). Genève : Droz, 2007, p. 179.

20. - Chiffre avancé par MICHEL, Jacques. La Guyane sous l'Ancien Régime. Paris : Harmattan, 1989.

21. - BOUCHER, Philip P. Les Nouvelles-Frances. Québec : Septentrion, 2005, p. 106. 
22. - BOUCHER, Philip P. Les Nouvelles-Frances. Québec : Septentrion, 2005, p. 134 : «Un simple tour d'horizon des publications françaises sur l'Amérique après 1763 montre clairement que les pertes coloniales de la France ne réduisirent en rien son intérêt pour le nouveau monde.

23. - WIMPFFEN, baron Alexandre Stanislas. Voyage à Saint-Domingue, 1788, 1789 et 1790. Paris : chez Cocherie, an V-1797, p. 113.

\section{RÉSUMÉS}

Parmi les rares publications consacrées à la construction des habitations à l'usage des colons aux Antilles et en Guyane, le manuel pratique du chevalier de Préfontaine intitulé "Maison rustique à l'usage des habitants de la partie de la France équinoxiale connue sous le nom de Cayenne » (Paris, Cl. J.B. Bauche, 1763) fait office d'exception. L'ouvrage est directement issu des expériences de terrain menées par l'auteur dans le quartier de Macouria où, mis aux arrêts pour insubordination en 1754, il se consacra gaiement à l'aménagement de sa propriété, l'habitation de la Félicité, tout en rédigeant un imposant vade-mecum pratique à l'attention des propriétaires terriens en Guyane et plus largement "dans les colonies en général». "Animé par un zèle patriotique du fond de son habitation ", Préfontaine détaille dans son manuel tous les aspects de l'architecture rurale coloniale s'intéressant non seulement à la disposition et à la construction d'une maison de maître mais également à ses nombreuses dépendances incluant cases des esclaves, magasin à vivre, cuisine en plein air, poulaillers et bâtiments d'exploitation tels les moulins sucriers et caféiers. Mais au-delà de cette contribution, l'ambition de l'ouvrage est bien de répondre à un dessein politique ambitieux : le peuplement de la Guyane par des colons blancs à la fin du XVII ${ }^{\mathrm{e}}$ siècle. Son ouvrage permet également de prendre la mesure de la propagande politique sous-jacente dans l'œuvre de Préfontaine dont le riant portrait agricole est publié quelques mois avant la désastreuse expédition de Kourou conduite par Chanvalon en 1764, qui se solda par la mort de 4000 nouveaux colons appâtés par la perspective de faire fortune dans la colonie.

Among the rare publications devoted to colonial housing in the West Indies and French Guyana, the manual titled "The Rustic House" (Maison rustique à l'usage des habitants de la partie de la France équinoxiale connue sous le nom de Cayenne) published in Paris by the chevalier de Préfontaine in 1763 stands out as an exception. Rather than being based on the on-site experiences Préfontaine led at La Felicité, his rural estate located in Macouria, a suburb of Cayenne, the book was written for all landowners of the colony and concerned more broadly "all the colonies in general". Préfontaine "Stirred by patriotic ardor from the bottom of his estate", energetically wrote a weighty manual on colonial rural architecture outlining the construction of the main house and its numerous outbuildings including the slave quarters, stores, hen house, open air kitchen and well as industrial buildings including sugar mills and coffee mills. Beyond his contribution to the field of rural architecture, Préfontaine's book responded to a more ambitious political scheme established at the end of the seventeenth century aiming to attract a white population of European settlers in the colony. Published a few months before the disastrous 1764 Kourou expedition led by Chanvallon, which caused the death of more than 4000 immigrants, Prefontaine's book reveals the structure of political propaganda underlying the deceptively pleasant portrait of the colony in the second half of the eighteenth century in the French colonies. 
INDEX

Mots-clés : littérature rurale, architecture coloniale, Guyane, XVIIIe siècle, édition

\section{AUTEURS}

\section{EMILIE D'ORGEIX}

Université Michel de Montaigne - Bordeaux 3 emilie.dorgeix@u-bordeaux3.fr

\section{CÉLINE FRÉMAUX}

Inventaire général du patrimoine culturel, Région Guyane celine.fremaux@cr-guyane.fr 